\title{
II. - APPRÉGIATION DE LA VALEUR COMMERCIALE
}

\author{
G. SCOTTI \\ avec la collaboration technique de C. Morne \\ Laboratoire central de l'Institut Technique des CérEales et des Fourrages, \\ 46, rue de la Clef, \\ Paris (5e) \\ RÉSUMÉ
}

La qualité des échantillons soumis aux divers traitements a été estimée selon les méthodes en usage dans le commerce.

Le séchage à $80^{\circ} \mathrm{C}$ immédiatement après récolte $\left(R_{g_{0}}\right)$ est le seul traitement qui ait permis d'obtenir, dans les deux essais, un mais commercialisable sans réfaction.

Tous les autres traitements ont provoqué des accidents qui entraînent le refus de tels lots à l'intervention, et en conséquence un écoulement difficile à bas prix.

\section{INTRODUCTION}

Le maïs grain destiné à l'autoconsommation est généralement séché à la ferme et fait rarement l'objet d'analyses ; sa valeur est estimée a posteriori par les performances réalisées par les animaux.

La plus grande partie de la récolte est cependant livrée et séchée dans les organismes stockeurs pour être mise à la vente. Le maïs est destiné à des industries de transformation comme l'alimentation animale, la semoulerie, l'amidonnerie ou à d'autres industries alimentaires.

Le commerce des grains étant libre, le contrat de vente est établi d'un commun accord entre acheteur et vendeur. Ils peuvent se référer à des contrats types, comme par exemple le Contrat de Paris, ou bien encore se reporter au décret de campagne de 1'Office National Interprofessionnel des Céréales (O. N. I. C.).

Le vendeur peut également présenter son maìs à l'organisme d'intervention (O. N. I. C.) qui est tenu de l'acheter s'il répond aux conditions du décret de campagne. Celui-ci définit notamment les critères et les normes d'analyse qui permettent de justifier éventuellement un refus ou d'établir le prix. En effet, le prix garanti 
ne s'applique qu'aux lots dont les résultats d'analyse s'inscrivent à l'intérieur de certaines tolérances.

Il reste entendu que le maïs refusé à l'intervention peut être cependant commercialisé de gré à gré mais son écoulement est alors très difficile et à des prix excessivement bas.

Les critères et les normes employés aussi bien dans les contrats commerciaux que pour l'intervention sont pratiquement identiques pour toutes les destinations et n'ont que des rapports lointains avec la valeur industrielle réelle mesurée par d'autres techniques plus spécifiques.

Le mais commercialisé représente actuellement plus de $77 \mathrm{p}$. xoo de la production contre 37 p. roo il y a $\mathrm{I} 2$ ans. Il faut donc bien tenir compte de cette " valeur commerciale " quitte à la faire modifier si l'on démontre qu'elle est très éloignée des réalités technologiques.

C'est cette valeur commerciale qui a été estimée pour les divers échantillons soumis à notre analyse.

\section{MATÉRIEL ET MÉTHODES}

- Dosage de l'eau: méthode AFNOR. Normes NF-Vo3-7or.

- Recherche des impuretés : la méthode employée répond aux directives de l'annexe I du règlement C. E. E. n ${ }^{\circ} 768 / 69$ du 22 avril 1969.

La recherche des impuretés se pratique par examen visuel sur une prise d'essai de Ioo grammes obtenue après homogénéisation et division de l'échantillon de laboratoire.

Les impuretés sont triées, pesées séparément et classées comme suit :

- grains avariés,

- grains germés,

- grains chauffés.

Les catégories d'impuretés " corps étrangers" et "grains brisés passant au travers du tamis à trous de $4,5 \mathrm{~mm}$ de diamètre " n'ont pas été portées sur les tableaux car elles étaient pratiquement inexistantes.

La catégorie grains brisés retenus par le tamis à trous de $4.5 \mathrm{~mm}$ de diamètre qui n'entre pas en ligne de compte pour le calcul du prix, est mentionnée en observation pour attirer l'attention sur la présence éventuelle d'un taux élevé de grains cassés, préjudiciable à la bonne conservation du maïs.

L'appréciation des résultats a été effectuée en fonction des normes du décret de campagne 1968-1969 qui définit les caractéristiques du maïs.

a) N.S.L.M. : non sain loyal et marchand. Le maïs n'est pas accepté par l'organisme d'intervention; son prix n'est plus garanti.

Il est caractérisé notamment par une humidité et un taux d'impuretés élevés.

- Humidité supérieure à 17,5 p. Ioo.

- + Io p. Ioo d'éléments autres que du maïs de qualité irréprochable.

- + to p. roo de grains germés.

- + 6 p. roo d'impuretés constituées par des grains.

b) Sans réfaction : le maïs est accepté par l'organisme d'intervention au prix garanti.

- L'humidité doit être comprise entre I4,5 et I5,4 p. 100 .

Les taux d'impuretés doivent être au maximum de :

- 4 p. Ioo pour les grains brisés,

- I p. roo pour les corps étrangers,

- 5 p. roo pour les impuretés constituées par des grains. 
c) Avec réfactions : le maïs est accepté par l'organisme d'intervention mais le prix est alors égal au prix garanti diminué du montant des réfactions. Celles-ci sont appliquées dans les limites de :

- 15,5 à r 7,5 p. Ioo pour l'humidité,

- 5 à 6 p. roo pour les impuretés constituées par les grains,

- I à ro p. roo pour les corps étrangers,

- 4 à ro p. roo pour les grains brisés.

\section{RÉSUL'TATS E'T DISCUSSION}

Les résultats des analyses effectuées sur les échantillons prélevés après traitements (stade III) sont présentés sur le tableau I. Ils montrent que le lot de maìs $\mathbf{R}_{\mathbf{8 0}}$ est le seul susceptible d'être commercialisé sans réfaction. Les autres lots ont subi diverses altérations mises en évidence par la présence d'un grand nombre de grains endommagés :

TABLEAU I

Analyse commerciale des échantillons de grain après traitement

\begin{tabular}{|c|c|c|c|c|c|c|c|}
\hline \multirow{2}{*}{ Échantillons } & \multicolumn{4}{|c|}{$\begin{array}{c}\text { Grains endommagés } \\
(\%)\end{array}$} & \multirow{2}{*}{$\begin{array}{c}\text { Valeur } \\
\text { commerciale }\end{array}$} & \multirow{2}{*}{$\begin{array}{c}\text { Aspect } \\
\text { général (1) }\end{array}$} & \multirow{2}{*}{$\begin{array}{c}\text { Grains } \\
\text { cassés } \\
\text { supérieurs à } \\
\text { 4, }, 5 \mathrm{~mm}(\%)\end{array}$} \\
\hline & Avariés & Chauffés & Germés & Total & & & \\
\hline $\begin{array}{l}\text { Témoin } \\
\text { récolte }\end{array}$ & 0,45 & 0 & 0 & 0,45 & sans réfaction & 5 & 0 \\
\hline $\begin{array}{l}\mathrm{R}_{80} \\
\mathrm{R}_{140}\end{array}$ & $\begin{array}{l}\text { traces } \\
0\end{array}$ & $\begin{array}{c}0 \\
11,70\end{array}$ & $\begin{array}{l}0 \\
\text { traces }\end{array}$ & $\begin{array}{l}0 \\
11,70\end{array}$ & $\begin{array}{l}\text { sans réfaction } \\
\text { N.S.L.M. }\left({ }^{\mathbf{2}}\right)\end{array}$ & $\begin{array}{l}5 \\
3\end{array}$ & $\begin{array}{l}0 \\
2,50\end{array}$ \\
\hline $\begin{array}{l}E_{80} \\
E_{140}\end{array}$ & $\begin{array}{l}6,65 \\
0\end{array}$ & $\begin{array}{r}0 \\
21\end{array}$ & $\begin{array}{l}11,90 \\
21,00\end{array}$ & $\begin{array}{l}18,55 \\
42,00\end{array}$ & $\begin{array}{l}\text { N.S.L.M. } \\
\text { N.S.L.M. }\end{array}$ & $\begin{array}{l}2 \\
1\end{array}$ & $\begin{array}{l}1,50 \\
1,35\end{array}$ \\
\hline $\begin{array}{l}F_{80} \\
F_{140}\end{array}$ & $\begin{array}{l}13,80 \\
13,40\end{array}$ & $\begin{array}{l}0 \\
5,50\end{array}$ & $\begin{array}{l}0 \\
0\end{array}$ & $\begin{array}{l}13,80 \\
18,80\end{array}$ & $\begin{array}{l}\text { N.S.L.M. } \\
\text { N.S.I.M. }\end{array}$ & $\begin{array}{l}3 \\
3\end{array}$ & $\begin{array}{l}0 \\
0,85\end{array}$ \\
\hline
\end{tabular}

(1) Aspect général : note subjective de 0 à 5 sur l'aspect général de l'échantillon.

(2) Non sain, loyal et marchand.

\section{à $\mathrm{I} 40^{\circ} \mathrm{C}$.}

- Grains chauffés (brûlés ou légèrement torréfiés) provenant des séchages

- Grains avariés dus aux effets des préstockages E et F ; il s'agit principalement de grains superficiellement moisis au cours du préstockage et recouverts d'une poussière noirâtre après séchage.

- Grains ayant germé par suite de l'échauffement du lot (entrent dans cette catégorie, les grains présentant un début de germination par gonflement du germe).

D’une façon générale, le séchage à température élevée $\left(140^{\circ} \mathrm{C}\right)$ accentue le pourcentage total de grains endommagés, surtout sur les maïs échauffés (E).

Les mêmes conclusions peuvent être tirées des résultats obtenus après une conservation de six mois (tabl. 2). Il faut cependant noter : 
- L'augmentation du taux de grains cassés retenus par le tamis à trous ronds de 4,5 $\mathrm{mm}$ de diamètre; ceci est dû aux manipulations effectuées sur les lots de maĩs au cours de la conservation (transilages, passage au nettoyeur, ensachage).

\section{TABLEAU 2}

Analyse commerciale des échantillons de grain après conservation (jusqu'au 8 juin I969)

\begin{tabular}{|c|c|c|c|c|c|c|}
\hline \multirow{2}{*}{ Échantillons } & \multicolumn{4}{|c|}{$\begin{array}{c}\text { Grains endommagés } \\
(\%)\end{array}$} & \multirow{2}{*}{$\begin{array}{c}\text { Valeur } \\
\text { commerciale }\end{array}$} & \multirow{2}{*}{$\begin{array}{c}\text { Grains } \\
\text { cassés } \\
\text { supérieurs à } \\
4,5 \mathrm{~mm}(\%)\end{array}$} \\
\hline & Avaries & Chauffés & Germés & Total & & \\
\hline $\begin{array}{l}\mathrm{R}_{80} \\
\mathrm{R}_{\mathbf{1 4 0}}\end{array}$ & $\begin{array}{l}0,40 \\
0\end{array}$ & $\begin{array}{r}0,45 \\
15,10\end{array}$ & $\begin{array}{l}0 \\
0\end{array}$ & $\begin{array}{r}0,95 \\
15,10\end{array}$ & $\begin{array}{l}\text { sans réfaction } \\
\text { N.S.L.M. }\end{array}$ & $\begin{array}{l}5,10 \\
5,30\end{array}$ \\
\hline $\begin{array}{l}E_{80} \\
E_{140}\end{array}$ & $\begin{array}{l}6,70 \\
0,80\end{array}$ & $\begin{array}{c}0 \\
22,15\end{array}$ & $\begin{array}{r}10,10 \\
9,85\end{array}$ & $\begin{array}{l}22,80 \\
32,80\end{array}$ & $\begin{array}{l}\text { N.S.L.M. } \\
\text { N.S.L.M. }\end{array}$ & $\begin{array}{l}3,45 \\
6,40\end{array}$ \\
\hline $\begin{array}{l}F_{80} \\
F_{140}\end{array}$ & $\begin{array}{l}8,95 \\
7,80\end{array}$ & $\begin{array}{l}0 \\
4,15\end{array}$ & $\begin{array}{l}0 \\
0\end{array}$ & $\begin{array}{r}8,95 \\
11,95\end{array}$ & $\begin{array}{l}\text { N.S.L.M. } \\
\text { N.S.L.M. }\end{array}$ & $\begin{array}{l}1,95 \\
3,40\end{array}$ \\
\hline
\end{tabular}

- La diminution du taux de grains avariés pour les traitements $F_{80}$ et $F_{140}$, ainsi que du taux de grains germés pour $\mathrm{E}_{80}$ et $\mathrm{E}_{140}$; on peut penser que les ventilations et les manipulations appliquées au grain ont permis une atténuation des signes d'altération : disparition partielle de moisissures superficielles et du gonflement du germe.

Il faut souligner que les méthodes employées ne visent uniquement qu'à déterminer la valeur commerciale dans le cadre des règlements techniques officiels en vigueur pour une campagne donnée. Les résultats ne sauraient être interprétés pour juger de l'état sanitaire du lot ou de sa valeur alimentaire ou industrielle.

\section{CONCI,USION}

Les échantillons des divers traitements, soumis à notre analyse, ont été examinés au moyen des méthodes en usage dans le commerce, et leur valeur exprimée d'après les barèmes officiels.

Les résultats ont montré, qu'à l'exception de l'échantillon $R_{80}$ dont la valeur marchande est intacte, tous les échantillons présentaient des signes divers d'altération profonde. De tels maïs sont refusés par l'organisme officiel d'intervention et sont fortement dépréciés dans le commerce. 


\section{SUMMARY}

\section{II - MEASUREMENTS OF THE COMMERCIAL VALUE}

The quality of the different samples has been estimated by the current rules and uses of the commercial trade. Kernels dried immediately after harvesting $\left(R_{80}\right)$ are the only suitable grains without drawbacks, for their use in commerce.

The measurements are based on the proportion of heated, germinated and damaged grains (mostly mouldy grains).

All the other maize processing methods produce a kernel damage and can reduce the commercial value of the grain. 